\title{
A Numerical Method for Determining the Coupling Strengths and Resonant Frequencies of a Nonperiodic Coupled Cavity Chain
}

\author{
M. Foley and T. Jurgens \\ Fermi National Accelerator Laboratory \\ P.O. Box 500, Batavia, IL 60510 USA
}

\begin{abstract}
Traditionally the coupling strengths and individual cavity resonant frequencies for a chain of coupled oscillators with periodic or biperiodic geometry have been calculated from the dispersion relation. A dispersion relation does not exist for a chain of coupled oscillators with nonperiodic geometry. A numerical procedure for estimating the unknown coupling strengths and resonant frequencies for individual elements of coupled oscillator chains has been developed and tested. This procedure has the novel capability that it is applicable to both periodic and nonperiodic structures.
\end{abstract}

\section{Introduction}

A chain of coupled linear accelerator cavities with biperiodic geometry is shown in Figure 1.

RESONATOR $R_{2 n-2} R_{2 n-1} R_{2 n} R_{2 n+1} R_{2 n+2}$

NUMBER

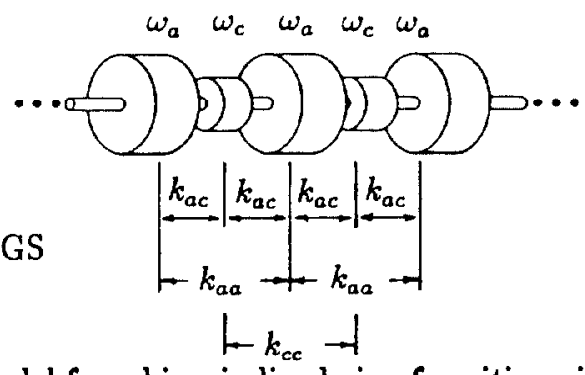

Figure 1: Model for a biperiodic chain of cavities with nearest neighbor and second nearest neighbor coupling

Investigation of the electrical properties of such a chain is accomplished by formulating a mathematical model consisting of a biperiodic chain of coupled oscillators (RLC circuits) with nearest neighbor and second nearest neighbor coupling[1], as shown in Figure 2.

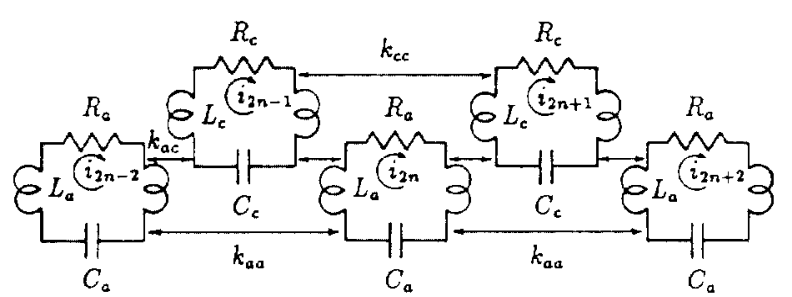

Figure 2: Coupled oscillator model for a biperiodic chain of cavities with nearest neighbor and second nearest neighbor coupling

The coupled equations generated from the model above [1] are of the form (assuming half cell termination):

$$
\begin{aligned}
I_{2 n}= & X_{2 n}\left[1+\frac{\omega_{a}}{j \omega Q_{a}}-\left(\frac{\omega_{a}}{\omega}\right)^{2}\right]+ \\
& \frac{k_{a c}}{2}\left(X_{2 n-1}+X_{2 n+1}\right)+ \\
& \frac{k_{a a}}{2}\left(X_{2 n-2}+X_{2 n+2}\right) \\
I_{2 n+1}= & X_{2 n+1}\left[1+\frac{\omega_{c}}{j \omega Q_{c}}-\left(\frac{\omega_{c}}{\omega}\right)^{2}\right]+ \\
& \frac{k_{a c}}{2}\left(X_{2 n}+X_{2 n+2}\right)+ \\
& \frac{k_{c c}}{2}\left(X_{2 n-1}+X_{2 n+3}\right)
\end{aligned}
$$

The quantities $I_{2 n}, I_{2 n+1}$ are forcing terms, $X_{2 n}$, $X_{2 n+1}$ are amplitudes, $\omega_{a}$ is the resonant frequency for individual accelerating cavities in the absence of coupling to their neighbors, $\omega_{c}$ is the resonant frequency for individual coupling cavities in the absence of coupling to their neighbors, $Q_{a}$ is the quality factor for accelerating cavities, and $Q_{c}$ is the quality factor for coupling cavities. 
There are $2 n+1$ solutions to the homogenous equations $\left(I_{2 n}=I_{2 n+1}=0\right)$ for the lossless case (high Q) of the form

$$
\begin{aligned}
X_{2 n} & =A \cos 2 n \phi_{q} \\
X_{2 n+1} & =B \cos (2 n+1) \phi_{q}
\end{aligned}
$$

where $\phi_{q}=\frac{\pi q}{2 N}, q=0,1, \ldots, 2 N$, provided that

$$
\begin{aligned}
k_{a c}^{2} \cos ^{2} \phi_{q}= & {\left[1-\left(\frac{\omega_{q}}{\omega_{q}}\right)^{2}+k_{a q} \cos 2 \phi_{q}\right] . } \\
& {\left[1-\left(\frac{\omega_{e}}{\omega_{q}}\right)^{2}+k_{c c} \cos 2 \phi_{q}\right] }
\end{aligned}
$$

$\phi_{q}$ is the phase shift per cavity in mode $q$, and $\omega_{q}, q=0, \ldots, 2 N$ are the frequencies of the normal modes of oscillation of the cavity chain. Equation (5) is the dispersion relation for a biperiodic chain of coupled oscillators with nearest and next nearest neighbor coupling.

It is important to note that the dispersion relation is not valid for a chain of nonperiodic coupled oscillators.

\section{Application of the Disper- sion Relation to a Side- Coupled Cavity Chain}

At FNAL the new LINAC side-coupled cavity sections have biperiodic geometry of the form shown in Figure 1. Based on the coupled oscillator model described in Section 1, the phase shift per cavity, $\phi_{q}$, is known for each normal mode frequency. Measurements are made using an HP network analyzer (NWA) of the corresponding normal mode frequencies. These values are used to generate a set of points $\left(\phi_{q}, \omega_{q}\right)$ in the $(\phi, \omega)$-plane. The coupling strengths $k_{a c}, k_{a a}$ and $k_{c c}$ and individual cavity resonant frequencies $\omega_{a}, \omega_{c}$ are determined from the dispersion curve by least squares fitting equation (5) to the measured points $\left(\phi_{q}, \omega_{q}\right)$ using an internal version of the LASL code DISPER[3].

\section{Matrix}

\section{Representation of the Cou- pled Oscillator Equations}

The homogenous form of equations $(1,2)$ for the case with no losses is given in vector-matrix format by

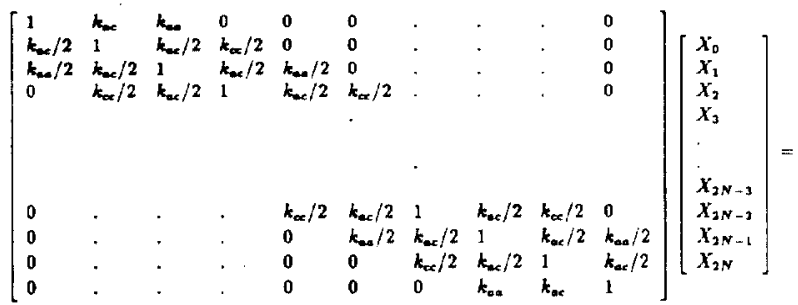

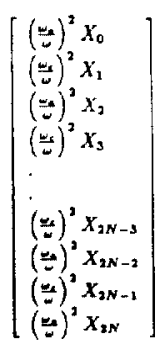

Equation $(B)$ is equivalent to equations $(1,2)$ with $I_{2 n}=I_{2 n+1}=0$ and $Q$ large.

Introducing the scale factor $\left(\frac{\alpha}{\omega_{i}}\right)^{2}, i=a, c$, equation (6) can be written in vector-matrix form as

$$
\mathbf{M X}=\lambda \mathbf{X}
$$

where $\lambda=\left(\frac{a}{\omega}\right)^{2}$. The eigenvalue problem ( 7 ) has $2 N+1$ solutions corresponding to the set of discrete eigenvalues $\lambda_{q}, q=0, \ldots, 2 N$. The corresponding normal mode frequencies are given by

$$
\omega_{q}=\frac{\alpha}{\sqrt{\lambda_{q}}}
$$

where $q=0, \ldots, 2 N$.

Consider again the problem of determining the coupling strengths and individual cavity resonant frequencies for a LINAC section. An alternative approach to the application of the dispersion relation is described below.

As before, a HP NWA is used to measure the normal mode frequencies, $\left(\omega_{q}\right)_{\text {measured }} q=0, \ldots, 2 N$, for the LINAC section under test. An initial estimate of the coupling strengths and resonant frequencies is made in order to initiate an iterative optimization algorithm. At each step in the execution of the algorithm the eigenvalue problem ( 7 ) is solved and corresponding normal mode frequencies $\omega_{q}, q=0, \ldots 2 N$ are calculated. A new step is made by choosing the coupling strengths and resonant frequencies to minimize the sum of the squares of the differences between the $\omega_{q}$ 's and the corresponding measured normal mode frequencies $\left(\omega_{q}\right)_{\text {measured }} q=0, \ldots, 2 N$. The optimization algorithm is implemented as a FORTRAN program with calls to two subroutines: (i) 


\begin{tabular}{|l|c|c|}
\hline & DISPER & Optimization \\
\hline$k_{a c} / 2$ & $2.657535510^{-2}$ & $2.657737710^{-2}$ \\
\hline$k_{a c} / 2$ & $-3.887846810^{-3}$ & $-3.873295110^{-3}$ \\
\hline$k_{c c} / 2$ & $3.928101810^{-4}$ & $3.994947910^{-4}$ \\
\hline$\omega_{\text {end }}$ & $806.32192 \mathrm{MHz}$ & $806.28166 \mathrm{MHz}$ \\
\hline$\omega_{a}$ & $804.64813 \mathrm{MHz}$ & $804.65951 \mathrm{MHz}$ \\
\hline$\omega_{c}$ & $807.88179 \mathrm{MHz}$ & $807.88676 \mathrm{MHz}$ \\
\hline
\end{tabular}

Table 1: Comparison of results from DISPER and Optimization Method for a LINAC side-coupled cavity section

EIGEN2, an eigenvalue solver [2], and (ii) DUNLSF, a least-squares minimization subroutine from the IMSL Mathematics Library. The minimization is carried out until the magnitude of the difference between two successive steps is less than a user specified tolerance.

The accuracy of the coupling strengths and normal mode frequencies determined by this approach, as compared to those determined by the traditional method using DISPER, is directly related to the level of precision obtained measuring the normal mode frequencies, $\left(\omega_{q}\right)_{\text {measured }}$.

Consider a typical LINAC side-coupled cavity section as shown in Figure 1 . The coupling strengths and individual cavity resonant frequencies for the section were determined from DISPER.

In order to set up a baseline test to determine the accuracy of the proposed procedure, assume the coupling strengths and individual cavity resonant frequencies are unknown. The normal mode frequencies for the section are available from NWA measurements. For the initial estimate $k_{a c}^{0} / 2=0.05, k_{a a}^{0} / 2=-0.005, k_{c c}^{0} / 2=0.0001, \omega_{a}^{0}=$ $805 M H z, \omega_{c}^{0}=805 M H z$, and $\omega_{\text {end }}^{0}=805 M H z$, application of the optimization procedure outlined above yields the results shown in Table 1 . Convergence required thirteen iterations. Further tests indicated that varying the initial estimate did not significantly effect either the results or the rate of convergence.

\section{Extension of the Optimiza- tion Method to Nonperiodic Structures}

A prototype LINAC side-coupled cavity module, consisting of two sections joined by bridge side cavities (bsc) and a bridge coupling cavity (bcc), is not a

\begin{tabular}{|c|c|}
\hline$k_{a, b c c} / 2$ & $-1.1082194 \quad 10^{-2}$ \\
\hline$k_{b c e, b, c} / 2$ & $3.5754545 \quad 10^{-2}$ \\
\hline$\omega_{b, c}$ & $798.79952 \quad \mathrm{MHz}$ \\
\hline$\omega_{b c c}$ & $809.58395 \mathrm{MHz}$ \\
\hline
\end{tabular}

Table 2: Coupling strengths and resonant frequencies for a LINAC side-coupled cavity module

biperiodic structure, and therefore the dispersion relation (5) is not valid. However, the optimization method described in Section 3 is applicable.

A vector-matrix representation of the coupled oscillator equations modeling the prototype LINAC module can be developed analogous to the procedure followed in Section 3. The parameters $k_{a c}^{i}, k_{a a}^{i}$, and $k_{c c}^{i}$ and $\omega_{a}^{i}, \omega_{c}^{i}$ are known for each of the two individual LINAC sections, $i=1,2$. The values of $k_{a, b c c}, k_{b c c, b, c}, \omega_{b, c}$ and $\omega_{b c e}$ are unknown, while $k_{b s c, b a c}$ is assumed to equal zero.

The numerical optimization procedure described in Section 3 was applied to determine $k_{a, b c c}, k_{b c c, b a c}, \omega_{b, c}$ and $\omega_{b c c}$ for such a nonperiodic structure. Convergence required ten iterations. The results are shown in Table 2.

\section{Conclusions}

A numerical procedure for estimating unknown coupling strengths and resonant frequencies for individual elements of coupled oscillator chains has been presented. The procedure has the novel capability that it is applicable to both periodic and nonperiodic structures.

\section{References}

[1] E.A. Knapp, B.C. Knapp, and J.M. Potter, "Standing Wave High Energy Linear Accelerator Structures," The Review of Scientific Instruments, Vol. 39, No. 7, pp. 979-991, July 1968.

[2] W.H. Press, B.P. Flannery, S.A. Teukolsky, and W.T. Vetterling, Numerical Recipes, Cambridge University Press, pp. 335-380, 1986.

[3] J. Crisp, "Fitting Frequency Measurements to the Dispersion Relation for a Doubly Periodic Chain of Resonators," FNAL Linac Upgrade Note LU-200, March 1993. 